\title{
578-THE RADIO-FREQUENCY DIELECTRIC PROPERTIES OF YEAST CELLS MEASURED WITH A RAPID, AUTOMATED, FREQUENCY-DOMAIN DIELECTRIC SPECTROMETER
}

\section{CHRISTINE M. HARRIS and DOUGLAS B. KELL *}

Department of Botany and Microbiology, University College of Wales, Aberystwyth, Dyfed, SY23 3DA (U.K.)

(Manuscript received March 5th 1983)

\section{SYMBOLS USED IN THIS PAPER}

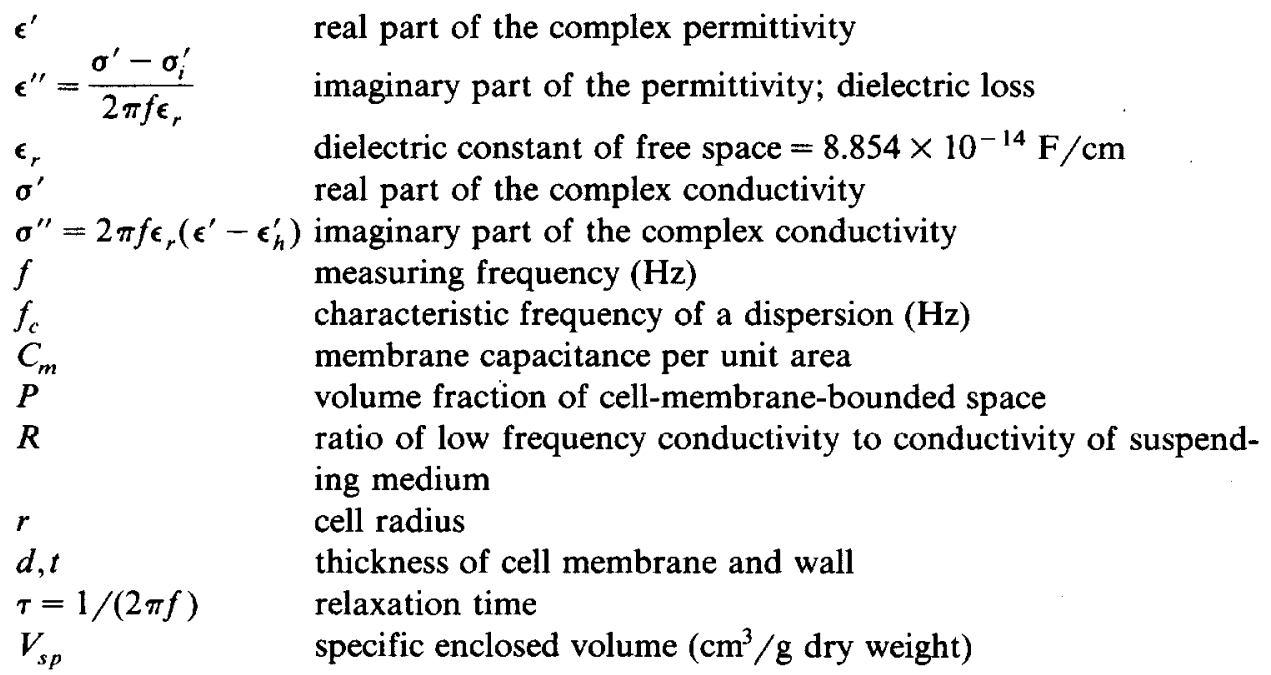

\section{Subscripts}

$\begin{array}{ll}l & \text { limiting value at low frequencies } \\ h & \begin{array}{l}\text { limiting value at high frequencies } \\ \text { suspending medium }\end{array}\end{array}$

\footnotetext{
* To whom all correspondence should be addressed. 


\section{SUMMARY}

(1) A computerized, rapid-scanning, frequency-domain dielectric spectrometer, capable of operating in the range $5 \mathrm{~Hz}-13 \mathrm{MHz}$, and based on commercially available components, is described.

(2) Measurements of the passive electrical properties of yeast cells in the range $0.01-13 \mathrm{MHz}$ confirm the well-documented existence of a pronounced Maxwell-Wagner $(\beta-)$ dispersion. The use of both complex permittivity (Cole/Cole) and complex conductivity diagrams indicates that dielectric relaxations centred at frequencies somewhat higher than those attainable contribute to the $\beta$-dispersion.

(3) The data obtained at different concentrations of the suspended phase are compared with the predictions of several suspension equations, some previously untested, and are found to be in excellent accord with them, even at volume fractions as high as 0.4 .

\section{INTRODUCTION}

A knowledge of the passive electrical properties of biological systems must underpin any significant understanding of the nature, role and mechanisms of bioelectrical phenomena [1-13]. To this end, a large body of experimental work, and in particular that of Schwan and.co-workers, has revealed that biological cell suspensions generally exhibit three major dielectric dispersions in the frequency range $10^{\circ}-10^{11} \mathrm{~Hz}$ (e.g. Refs. 1, 3-6, 11, 12). The $\alpha$-dispersion is seen at audio frequencies, and is largely ascribable to counterion relaxation tangential to charged surfaces [e.g. Refs. 14-16], while the $\beta$-dispersion, centred in the radio-frequency range, is dominated by a Maxwell-Wagner effect at the interface between the suspending medium and the poorly conducting plasma (cytoplasmic) membrane. The $\gamma$-dispersion, which will not here concern us further, takes place at microwave frequencies, and is caused by the relaxation of small dipolar species.

Since our own interests lie in particular in understanding the nature of the electrical phenomena implicated in processes such as electron transport phosphorylation (e.g. Refs. 13, 17, 18) catalysed by a wide variety of biological membranes, we have sought to develop a system capable of measuring rapidly the dielectric properties of biomembrane systems, under a variety of physiological conditions, in the audio- and radio-frequency ranges.

Traditionally, biological impedance measurements have been effected using manual bridge-balancing techniques (e.g. Refs. 1, 5, 6, 19-22), but the long measuring times required to obtain spectra have generally necessitated the use of dormant, non-metabolizing cells, so as to obviate any metabolism-induced changes in suspension conductivity as the frequency range of interest was scanned. However, the last 5 or 10 years have witnessed the appearance of both in-house and commercially available automated, digital impedimetric instrumentation of great power, speed and accuracy. Thus, Pilla and co-workers have developed time-domain three- and four-electrode systems which exploit an ultrafast potentiostat and digital Laplace transformation of the measured response [e.g. Refs. 7, 23, 24]. Coster, Smith and co-workers have described a low-frequency four-electrode computerized impedimetric system especially suited to work with artificial bilayer membrane systems 
[25-27], and a number of groups have exploited a variety of cognate methodologies in a plethora of elegant and detailed physical [28-30], electrochemical [31-36], biophysical [37-42] and neurophysiological [43-47] studies.

The purpose of the present paper is to describe our own realization of an automated, rapid-scanning, frequency-domain dielectric spectrometer, capable of operating in the range $5 \mathrm{~Hz}-13 \mathrm{MHz}$. We illustrate the utility of the system by presenting measurements of the Maxwell-Wagner [ $\beta$-] dielectric dispersion of intact yeast cells, an organism chosen because of its spherical nature and narrow size distribution.

\section{EXPERIMENTAL}

\section{Impedimetric apparatus}

Admittance measurements (of the equivalent parallel conductance and capacitance of the sample of interest) were made using a Hewlett-Packard 4192A impedance analyser controlled, via an interface bus conforming to the IEEE-488 standard, by a Hewlett-Packard HP85 computer ( 32 kilobytes core memory). Data were stored in the computer memory for transformation into complex plane diagrams (see below), and were displayed on the screen of the computer before being plotted, together with any desired equations, on a Hewlett-Packard Model 7225A plotter. Computer programs were written by the authors in BASIC. A block diagram of the apparatus is given in Fig. 1 and pertinent methodological details in the appropriate sections.

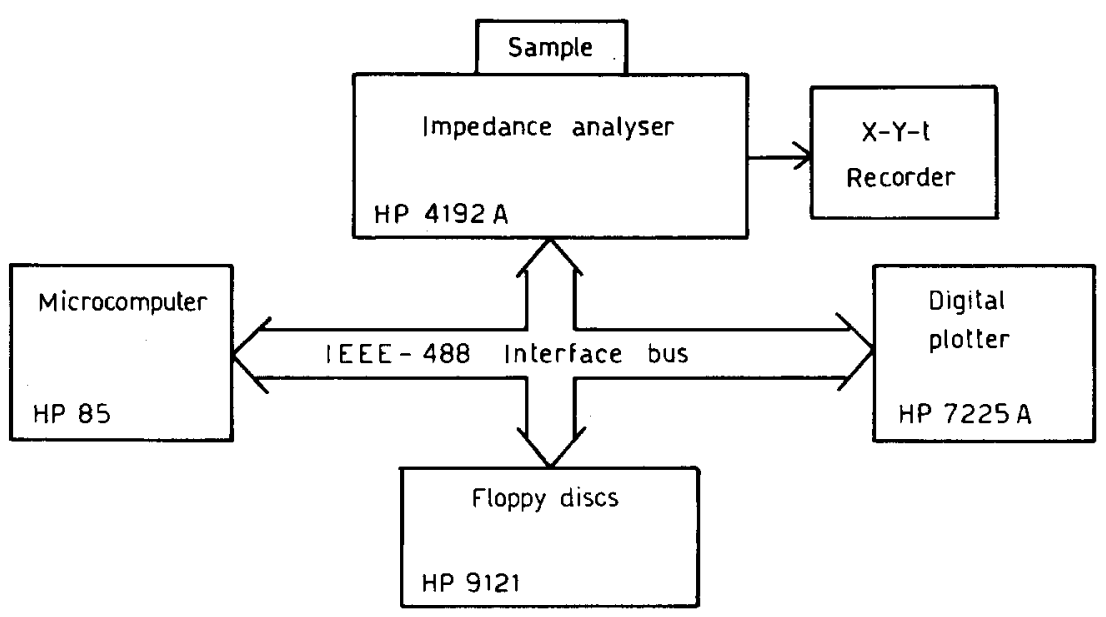

Fig. 1. Block diagram of the dielectric spectrometer. 


\section{Cell and electrode configuration}

The cell used in the present experiments was of the pin type described by Schwan $[19,48]$. Ti electrodes led directly to the (Model 16047A) test fixture supplied with the impedance analyser. The electrodes were cleaned in $1 M \mathrm{HCl}$ and plated with $\mathrm{Pt}$ black in situ using the usual Kohlrausch solution [19], a Pt foil counterelectrode, an $\mathrm{Ag} \mid \mathrm{AgCl}$ double-junction reference electrode and a Princeton Applied Research $174 \mathrm{~A}$ potentiostat. The plating voltage was adjusted to give a current density of 10 $\mathrm{mA} / \mathrm{cm}^{2}$ and plating was continued for two hours. Visual and microscopic inspection showed that an adequate coat of Pt black had been deposited, and admittance measurements using $\mathrm{KCl}$ solutions indicated that electrode polarization had been reduced to a level acceptable for the present purposes. Electrodes were shorted and immersed in distilled water when not in use, as suggested [19]. Measurements were carried out at room temperature $\left(20^{\circ} \mathrm{C}\right)$.

\section{Calibration and determination of cell constant}

As discussed in extenso by Schwan [1,19] and by Grant et al. [5], two major problems may beset those who would measure biological impedances in the audioand radio-frequency ranges. At low frequencies, electrode polarization artefacts may assume prominence, while at high frequencies parasitic circuit elements, notably a series resistance, lead inductance and stray capacitances, will tend to distort the true readings. Each of these potential problems is exacerbated as the conductivity of the suspension of the system is increased. Since one of the main purposes of the present work was to develop a rapid bioimpedance measuring system, one design goal was to minimize the number of corrections made to the bridge readings. In the present experiments, using media of relatively low specific conductance, it was found adequate to use the capacitance and conductance measurements as obtained. There was a slight, but measurable, decrease in the apparent conductance both of standard resistors and of ionic solutions at frequencies above approximately $4 \mathrm{MHz}$ in the present range of conductances studied; however, this decrease amounted to only approximately $1.5 \%$ of the true reading at $10 \mathrm{MHz}$. No corrections were therefore made to these readings to obtain the true readings in the presence of biological materials. In the 4192 instrument a zero open adjustment permits the automatic correction of measured data for simple strays and residuals, and this was employed using the empty cell. The real part of the permittivity of the sample was thus obtained [49] from the formula.

$\epsilon^{\prime}=\epsilon_{A q}^{\prime} \frac{C_{s}}{C_{A q}}$

in which $\epsilon_{A q}^{\prime}$ is the permittivity of water at the measurement temperature, while $C_{s}$ and $C_{A q}$ are respectively the capacitances of the sample and of distilled water measured after (automated) correction of the readings, via the zero open feature, for the capacitances of the empty cell, all such calibrations being carried out at $1 \mathrm{MHz}$. 
The cell constant obtained via the factor $\epsilon_{A q}^{\prime} / C_{A q}$ was checked by measuring the capacitance of the cell filled with methanol $\left(\epsilon^{\prime}=33.64\right.$ at $\left.20^{\circ} \mathrm{C}\right)$, and was found to be accurate throughout the frequency range studied to better than $1 \%$. Conductivities were obtained using the cell constant derived from capacitance measurements and the measured conductances, and were checked using $\mathrm{KCl}$ solutions of known conductivity. The cell constants calculated using the two methods were in excellent agreement with each other, and were approximately $0.62 \mathrm{~cm}^{-1}$.

\section{Biological material}

Baker's yeast (Saccharomyces cerevisiae) was obtained from the Distiller's Company Ltd. and stored at $4^{\circ} \mathrm{C}$ before use. No changes in dielectric properties were observed during periods of storage up to 10 days. Before use, cells were dispersed as a $20 \%(w / V)$ suspension, and washed three times, in a medium containing $0.2 \mathrm{M}$ sorbitol, $1 \mathrm{~m} M$ magnesium sulphate, $0.5 \mathrm{~m} M$ sodium EDTA and $5 \mathrm{~m} M$ tris tricinate $\mathrm{pH}$ 7.0. Cell densities in the final stock suspensions were in the range $130-160 \mathrm{mg}$ dry weight $/ \mathrm{cm}^{3}$.

\section{RESULTS AND DISCUSSION}

The $\beta$-dispersion of yeast cells, and its representation in complex plane plots

A typical dielectric spectrum of a yeast cell suspension in the range $10 \mathrm{kHz}-13$ $\mathrm{MHz}$ is shown in Fig. 2. The general form of this spectrum is in substantial agreement with previously published data obtained by others [50,53], although we find no evidence for an $\alpha$-dispersion in the frequency range studied. A feature to be stressed is that we were able to obtain the entire dispersion curve, with 20 frequency points per decade, in under 30 seconds, thus obviating the need [52,53] to add agar to the suspensions.

When data for a complete dispersion curve are not available, it is usual to analyse such dispersions by means of the complex permittivity (Cole/Cole) [54] diagram, in which $\epsilon^{\prime \prime}=\left(\sigma^{\prime}-\sigma_{l}^{\prime}\right) /\left(2 \pi f \epsilon_{r}\right)$ is plotted against $\epsilon^{\prime}$. We have chosen to utilize two complementary complex plane plots, namely the complex conductivity (admittance) plot [55], of $\sigma^{\prime \prime}=2 \pi f \epsilon_{r}\left(\epsilon^{\prime}-\epsilon_{h}^{\prime}\right)$ versus $\sigma^{\prime}$, as well as the more common Cole/Cole plot. The virtue of this approach, as noted for instance by Grant [55], Daniel [21] and Stoy et al. [11] is that the weightings of the two representations are substantially different. Thus, the Cole/Cole plot is extremely sensitive to the choice of the value of $d . c$. conductivity $\left(\sigma_{i}^{\prime}\right)$, which must be substracted from the observed conductivities in the calculation of the dielectric loss. On the other hand, the complex conductivity plot gives excellent values for the d.c. (or low-frequency) conductivity, which are independent, over a substantial range, of the values chosen for the high-frequency permittivity $\left(\epsilon_{h}^{\prime}\right)$. These points are illustrated in Figs. 3 and 4, using the data given in Fig. 2.

Figure 3 shows complex conductivity plots of the data in Fig. 2. It is evident that 


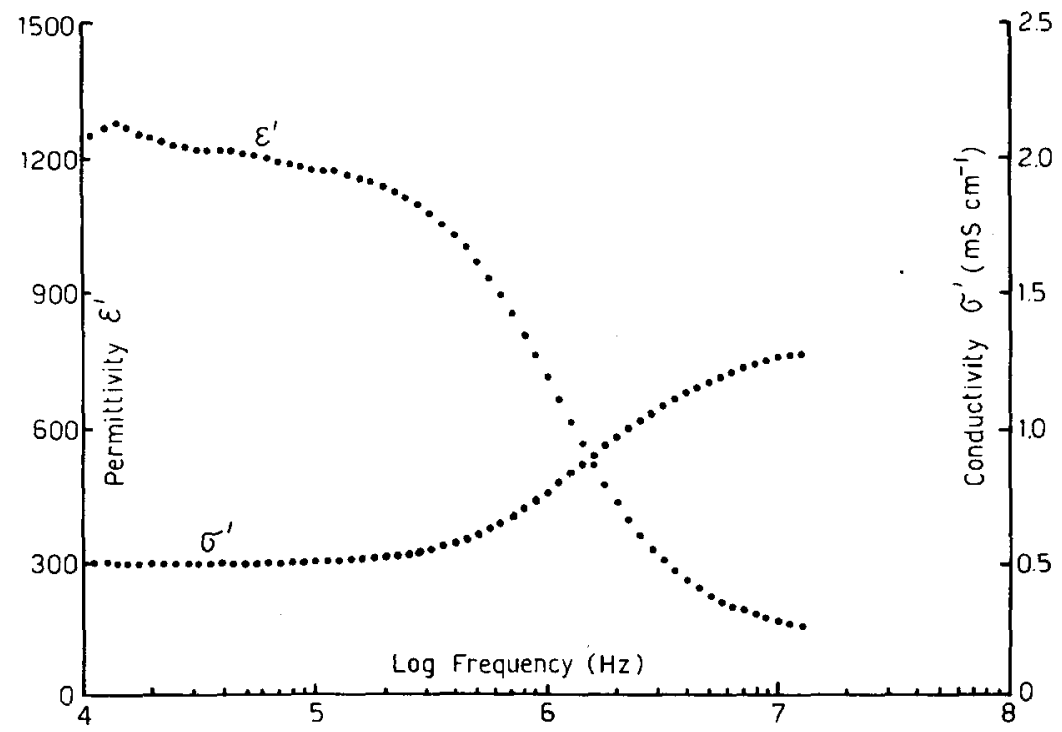

Fig. 2. A typical dielectric spectrum of intact yeast cells suspended as described in the Experimental section. Yeast cell concentration $=162 \mathrm{mg}$ dry $\mathrm{wt} / \mathrm{cm}^{3}$.

the choice of a value of $\epsilon_{h}^{\prime}$ equal to that of the suspending medium (approx. 80) leads to a complex conductivity plot that follows a semicircular locus for only one-half of the dispersion, although the $\sigma_{i}^{\prime}$ value obtained by extrapolation of the plot to the abscissa is independent of the choice of $\epsilon_{h}^{\prime}$ between values of 80 and 130 (Fig. 3). In contrast, the Cole/Cole representation is evidently highly sensitive to the choice of

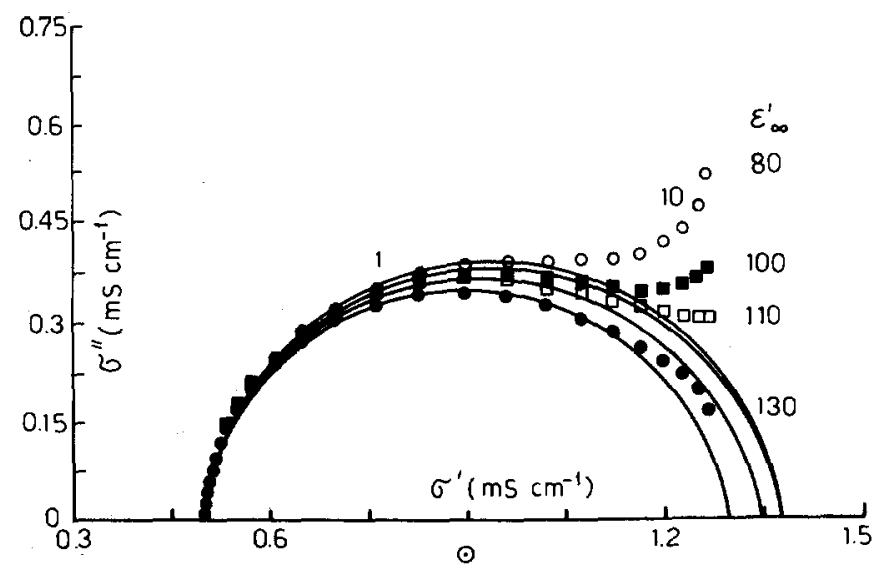

Fig. 3. Complex conductivity plot of yeast cell dielectric spectra. The data in Fig. 2 are plotted in the form of a complex conductivity diagram using the arbitrarily chosen values of $\epsilon_{h}^{\prime}$ indicated. Frequencies in MHz. 


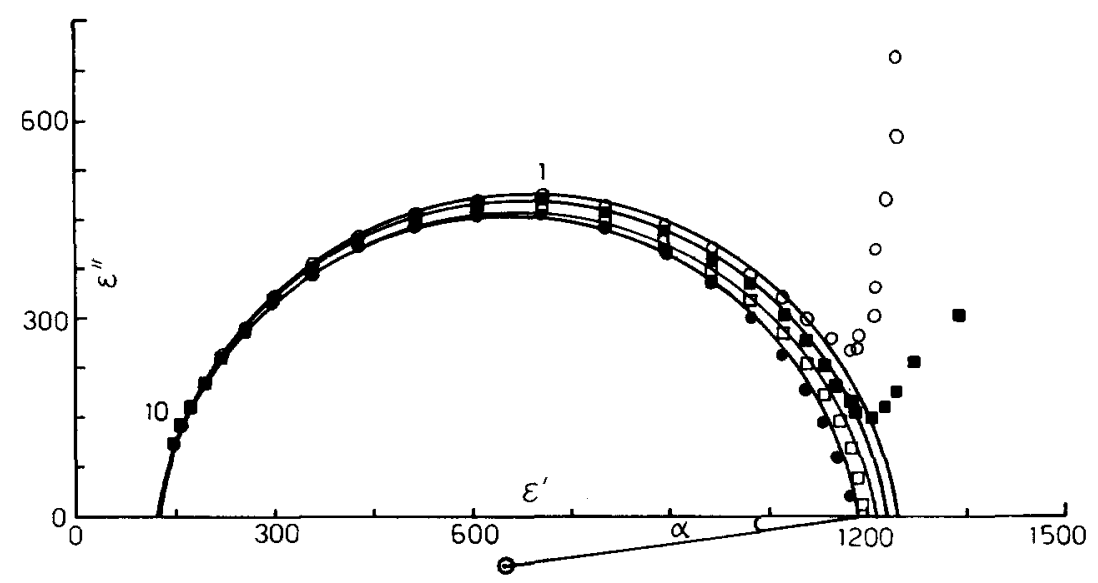

Fig. 4. Complex permittivity (Cole/Cole) plot of yeast cell dielectric spectra. The data in Fig. 2 are plotted using the following values of $\sigma_{1 \mathrm{im}}^{\prime}$ : (ब) $0.510 \mathrm{mS} / \mathrm{cm} ;(\square) 0.505 \mathrm{mS} / \mathrm{cm} ;(\square) 0.500 \mathrm{mS} / \mathrm{cm} ;(0)$ $0.495 \mathrm{mS} / \mathrm{cm}$. Frequencies in $\mathrm{MHz}$.

$\sigma_{i}^{\prime}$, the best fit being taken as that which best conforms to a semicircular locus while avoiding any negative $\epsilon^{\prime \prime}$ values. In fact an inaccuracy of $n \%$ in the choice of $\sigma_{l}^{\prime}$ leads to an inaccuracy of approximately $2 n \%$ in the magnitude of the dielectric dispersion estimated from the complex permittivity plots. The $\epsilon_{l}^{\prime}$ and $\epsilon_{h}^{\prime}$ values were then read from the Cole/Cole plot, and, if desired, another complex conductivity plot could be obtained using the $\epsilon_{h}^{\prime}$ value derived from the Cole/Cole plot. In this way, the dielectric parameters $\sigma_{l}^{\prime}, \sigma_{h}^{\prime}$, $\epsilon_{l}^{\prime}$ and $\epsilon_{h}^{\prime}$ could be conveniently extracted from measured dispersion curves such as those shown in Fig. 2.

\section{Comparison of observed dielectric behaviour with some suspension equations}

The quality of the data obtained prompted us to compare the dielectric behaviour observed with that predicted on the basis of various so-called suspension equations that have appeared in the literature. A number of authors have considered the relationship between $\sigma_{l}^{\prime}$ and $\sigma_{o}^{\prime}$, where $\sigma_{o}^{\prime}$ is the conductivity of the suspending medium. A widely used equation (e.g. Refs. 56-58), dating from the nineteenth-century work of James Clerk Maxwell and Lord Rayleigh, is

$R=\frac{1-P}{1-P / 2}$

where $R$ is the ratio $\sigma_{l}^{\prime} / \sigma_{o}^{\prime}$ and $P$ the volume fraction of the suspended phase. This equation assumes that a low concentration $(P<0.2)$ of non-conducting particles is present. The equation

$R=(1-P)^{3 / 2}$ 
based on the Bruggeman approximation was considered [57,59] to be a better representation for imperfectly spherical particles, while for volume fractions exceeding 0.2 , the equation

$R=\frac{8[2 P(1-P)]}{(4+P)(4-P)}$

was proposed [60]. Finally, in a study of yeast cells, Asami et al. [52] proposed

$R=\frac{2(1+2 \phi)(1-V) x+2(1-\phi)(2+V)}{2(1-\phi)(1-V) x+(2+\phi)(2+V)}$

where $x$ is the ratio of cell wall conductivity to suspending medium conductivity, $\phi$ the volume fraction including the cell wall, $D$ the inner diameter of the cell, $d$ and $t$ the thicknesses of the cell membrane and cell wall respectively and

$V=\left(\frac{D+2 d}{D+2 d+2 t}\right)^{3}$

The cited authors [52] found, for an inner-membrane-bounded cell diameter of $3.8 \mu \mathrm{m}$ and a cell wall thickness of $0.25 \mu \mathrm{m}$ that a cell wall conductivity equal to that of the suspending medium gave an adequate fit to the data. The curves of equations (1-4), the latter using the above values of $D$ and $t$, and values of $10^{-2} \mu \mathrm{m}$ for $d$, and 1 for $x$, are plotted together in Fig. 5. The low-frequency conductivity values of a

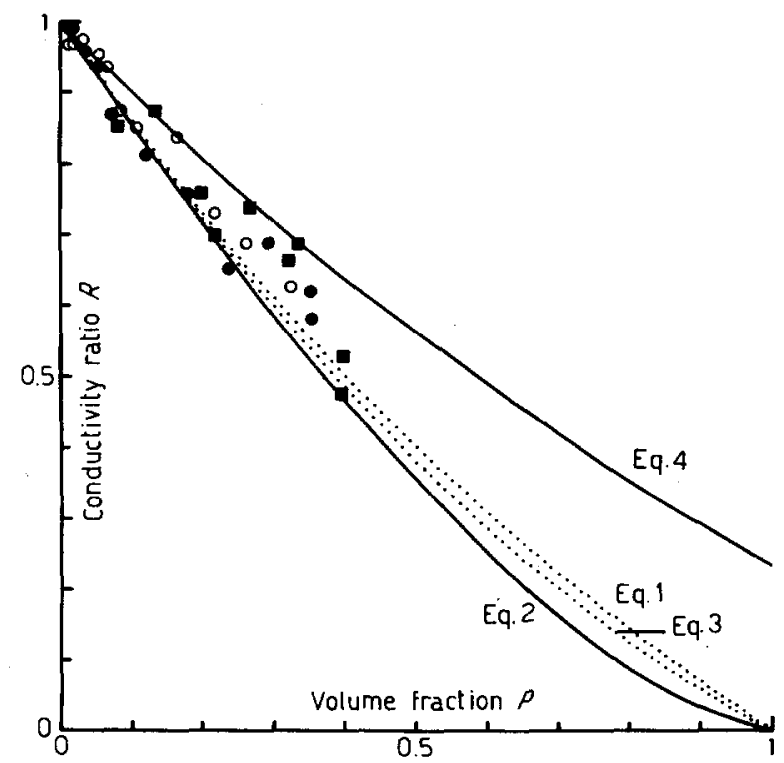

Fig. 5. Effect of volume fraction of yeast cells on low-frequency conductivity; $\sigma_{i}^{\prime}$ values were obtained from complex conductivity plots such as those in Fig. 3. Data are plotted as the ratio of $\sigma_{l}^{\prime}$ to $\sigma_{o}^{\prime}$ versus the volume fraction obtained according to the procedure, based on $\epsilon_{l}^{\prime}$ measurements, described in the text. The different symbols represent various dilutions of three separate yeast cell preparations. $(-\ldots, \cdots \cdots)$ Plots of the equations indicated. 
number of yeast cell suspensions were determined from complex conductivity diagrams as described above, and the conductivities of their suspending media determined after removing the yeast cells by centrifugation in an Eppendorf microfuge. It is evident (Fig. 5) that a variable amount of leakage of ionic constitutents from the cells introduced a degree of scatter into these data too great to distinguish the accuracy of the proposed models, which, it may in any event be noted, follow, with the exception of that of Asami et al. [52], rather similar loci. It may be concluded that any available suspension equations based solely upon conductivity measurements may be fitted to yeast cell data within the precision of the measurements possible. We therefore consider suspension equations based upon (complex) permittivity measurements.

\section{Low-frequency permittivity data}

The equation most commonly used to describe the low-frequency permitivity of cell suspensions (e.g. Refs. 1, 11, 49, 61), based upon the assumption that the cell membrane is essentially non-conducting at low frequencies, is

$\epsilon_{l}^{\prime}=\epsilon_{h}^{\prime}+\frac{9 P C_{m} r}{4 \epsilon_{r}}$

where $r$ is the cell radius (neglecting the cell wall) and $C_{m}$ the specific capacitance of the cell membrane (per $\mathrm{cm}^{2}$ ). Equation (6) permits the physically unreasonable assumption that the membrane volume is a negligible fraction of the total volume (and also that cells do not shield each other), and Schwan et al. [58] therefore extended equation (6) as follows:

$\epsilon_{l}^{\prime}=\epsilon_{o}^{\prime} \frac{(1-P)}{(1+P / 2)}+\frac{9 r C_{m}}{4 \epsilon_{r}}\left[\frac{P}{(1+P / 2)^{2}}\right]$

However, the cited authors were not able adequately to test equation (7) in their phospholipid vesicle system since the magnitude of the Maxwell-Wagner dispersions obtained were extremely small. This is because such a dielectric increment depends strongly on both the volume fraction and the radius of the suspended particles, and the diameter of the phospholipid vesicles used was very small [13.5 nm]; indeed, the accessible volume fractions used in the above study (Ref. 58, cf. also Ref. 62), up to 0.14 , were such that $\epsilon^{\prime}$, was for practical purposes linear with the right-hand side of equation (7). Thus, equation (7) has not yet been tested on a biomembrane vesicle suspension of high volume fraction.

The strategy we adopted, in assessing the consonance of equation (7) with experimental data, was to assume that the dry weight and the radius $(1.9 \mu \mathrm{m})$ of the cells could be measured accurately. The volume fraction, $P$, could then be obtained from the known yeast concentration, $C$, in $\mathrm{mg}$ dry weight $/ \mathrm{cm}^{3}$, provided that the specific enclosed volume of the cells, $V_{s p}$, in $\mathrm{cm}^{3} / \mathrm{g}$ dry weight, was known, since $P=V_{s p} C / 1000$. However, the sensitivity of the plot of equation (7), for a chosen $C_{m}$ 


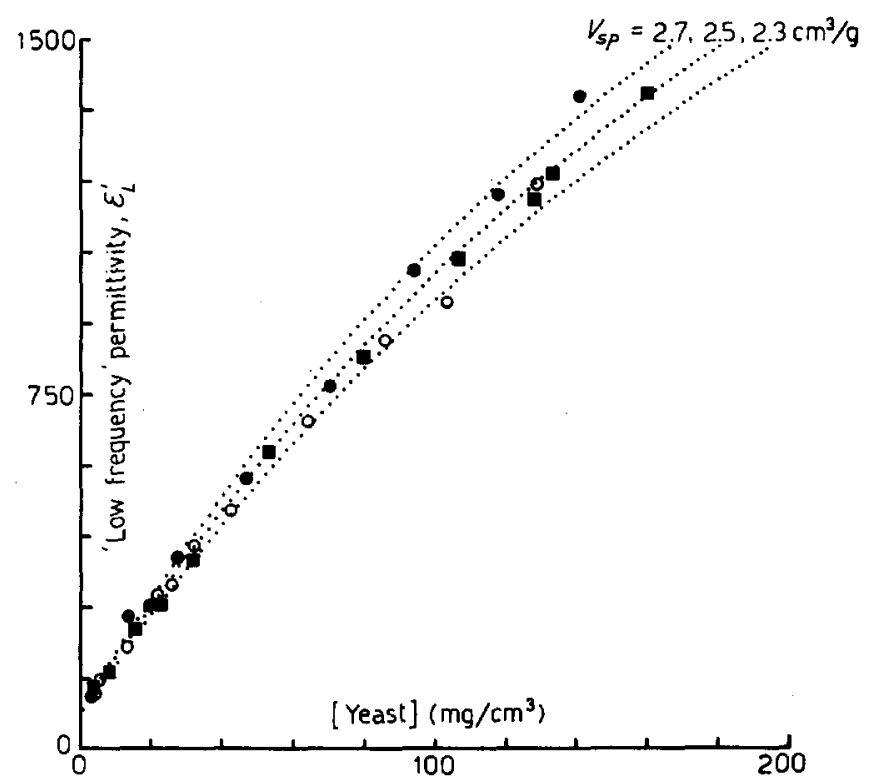

Fig. 6. Effect of yeast cell concentration on low-frequency permittivity. The $\epsilon_{i}^{\prime}$ values were obtained from complex permittivity diagrams such as those in Fig. 4, using three separate stock suspensions of yeast at

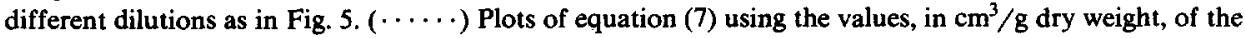
specific enclosed volume indicated. For further details, see text.

value of $1 \mu \mathrm{F} / \mathrm{cm}^{2}[2,12,52,63]$, to the specific volume chosen, exceeds that of the experimental accuracy in obtaining specific enclosed volumes using even the best isotope dilution methods (see e.g. Refs. 64, 65). We therefore display, in Fig. 6, the low-frequency permittivity as a function of yeast concentration together with plots of equation (7) using various choices of the specific enclosed volume. It is clear that equation (7) provides an excellent fit to the observed data using a specific enclosed volume of $2.5 \mathrm{~cm}^{3} / \mathrm{g}$ dry weight, and that the present approach provides a convenient method for the estimation of $V_{s p}$.

High-frequency permittivity values, as obtained by extrapolation of complex permittivity diagrams

The $\epsilon_{h}^{\prime}$ values obtained by plotting the dielectric dispersions in the complex permittivity plane, and extrapolating the semicircular loci to the abscissa, are plotted as a function of yeast cell concentration in Fig. 7. It is evident that there is a high-frequency dielectric increment $\left(\epsilon_{h}^{\prime}-\epsilon_{\mathrm{H}_{2} \mathrm{O}}^{\prime}\right)$ amounting to some 25 dielectric units per $100 \mathrm{mg}$ dry weight yeast $/ \mathrm{cm}^{3}$, a feature not noted in earlier studies (e.g. Refs. 52, 53), but especially evident in complex conductivity plots (Fig. 3). However, the present result is to be expected from a consideration of the structure of yeast 


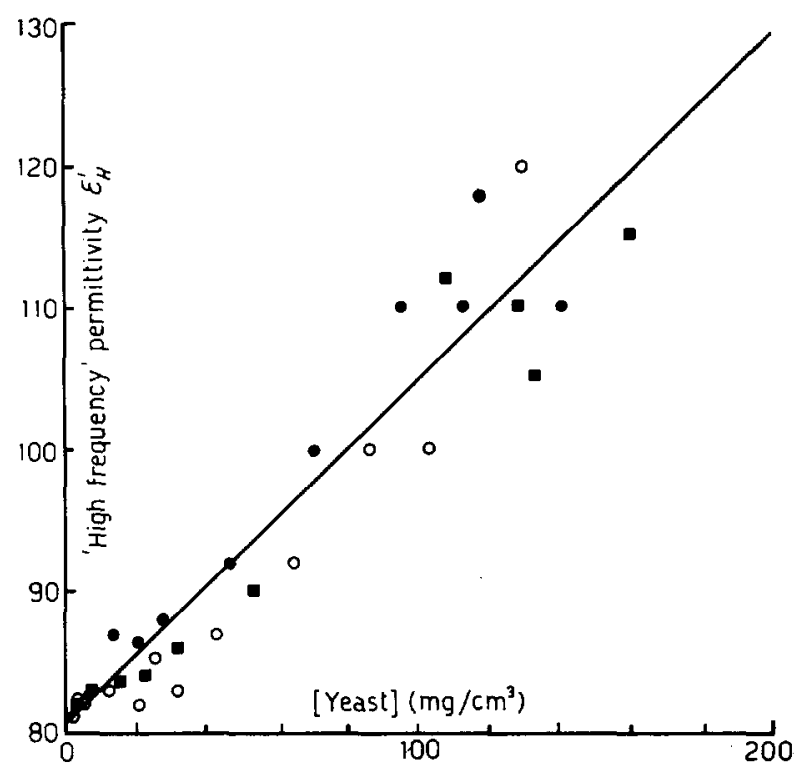

Fig. 7. Effect of yeast cell concentration on high-frequency permittivity. Data were obtained from complex permittivity plots using the samples of Figs. 5 and 6.

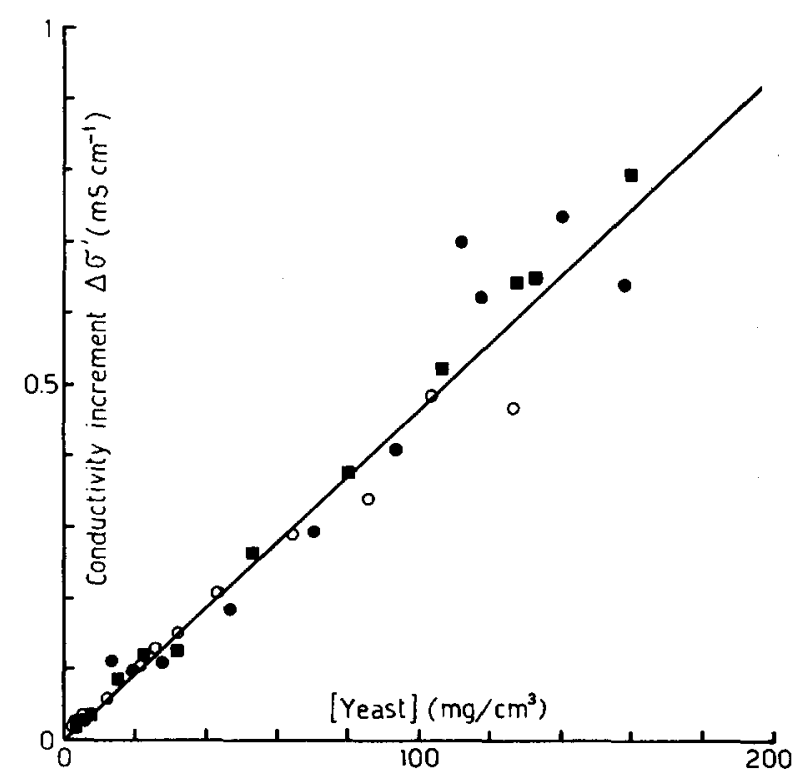

Fig. 8. Effect of yeast cell concentration on the conductivity increment. Data were obtained from complex conductivity plots, such as those in Fig. 3, using the samples of Figs. 5-7. 
cells [66] and the empirical nature of the Cole/Cole analysis of the complex permittivity plot [11]. Thus, we have so far treated the yeast cell membrane as the sole component likely, via the Maxwell-Wagner effect, to contribute to the $\beta$-dispersion. However, the dispersion both of the intracellular proteins, bound water and mitochondrial membranes may be expected to take place with critical frequencies of perhaps $10-100 \mathrm{MHz}$ (e.g. Refs. 11, 67), so that their dispersion, though not their existence, will go unnoticed if measurements are confined to the frequency range of that in the present study. It is clear that, being a property of the yeast cells, the apparent, net high-frequency dielectric increment (we omit from explicit consideration the contribution of any coexistent but smaller high-frequency dielectric decrement) should be a linear function of the yeast cell concentration, as observed (Fig. 7).

\section{The conductivity increment}

The conductivity increment $\left(\sigma_{h}^{\prime}-\sigma_{l}^{\prime}\right)$ of the yeast $\beta$-dispersion, obtained from complex conductivity plots such as those in Fig. 3, is plotted as a function of the yeast cell concentration in Fig. 8. The relationship is evidently well described by a straight line, as to be expected (e.g. Refs. 8, 11).

\section{Critical frequency and distribution of relaxation times}

In the Cole/Cole analysis of the complex permittivity diagram, the angle $\alpha$ (see Fig. 4) is taken to indicate the distribution of relaxation times contributing to the observed dispersion. This value is only 0.09 in the experiment of Fig. 4, and analysis of 30 separate observations, corresponding to the data in Fig. 6, gave a value for $\alpha$ of $0.146 \pm 0.023$ (mean \pm s.e.m.), indicating that the spread of relaxation times was indeed rather small (cf. Ref. 51), and much less than that observed with a number of bacteria [68]. The critical frequencies determined from complex permittivity and complex conductivity plots respectively, were, for the same data, $803 \pm 115 \mathrm{kHz}$ and $990 \pm 175 \mathrm{kHz}$, corresponding to a (geometric) mean critical frequency of $890 \mathrm{kHz}$ and a relaxation time $\tau[=1 /(2 \pi f)]$ of $0.18 \mu \mathrm{s}$, a value well within the range usually reported for the $\beta$-dispersion of microorganisms of this size, suspended in a medium of the present conductivity (approx. $0.67 \mathrm{mS} / \mathrm{cm}$ ), and consistent with an internal conductivity of approx. $2.3 \mathrm{mS} / \mathrm{cm}$, as also reported by others [52,53].

\section{CONCLUSIONS}

The apparatus described in the present paper constitutes a rapid and convenient system for determination of the passive electrical properties of biological materials. Measurements with intact yeast cells indicate that processes other than a Maxwell-Wagner effect contribute to the high-frequency end of the $\beta$-dispersion, but that otherwise the classical suspension equations provide an excellent fit to the data obtained. The present system would seem to possess a rapidity adequate to 
assess the dielectric properties of metabolizing biological systems under different physiological conditions.

\section{ACKNOWLEDGEMENTS}

We thank the Science and Engineering Research Council, ICI plc and the Royal Society for financial support.

\section{REFERENCES}

1 H.P. Schwan, Adv. Biol. Med. Phys. 5 (1957) 147.

2 K.S. Cole, Membranes, Ions and Impulses, University of California Press, Berkeley, 1972.

3 E.L. Carstensen and R.E. Marquis, in Spores VI, P. Gerhardt, R.N. Costilow and H.L. Sadoff (Editors), American Society for Microbiology, Washington, 1975, p. 563.

4 O.F. Schanne and E.R.P. Ceretti, Impedance Measurements in Biological Cells, Wiley, Chichester, 1978.

5 E.H. Grant, R.J. Sheppard and G.P. South, Dielectric Behaviour of Biological Molecules in Solution, Oxford University Press, London, 1978.

6 R. Pethig, Dielectric and Electronic Properties of Biological Materials, Wiley, Chichester, 1979.

7 A.A. Pilla, in Bioelectrochemistry, H. Keyzer and F. Gutmann (Editors), Plenum Press, New York, 1980, p. 353.

8 H.P. Schwan and K.R. Foster, Proc. I.E.E.E., 68 (1980) 104.

9 M.A. Stuchly and S.S. Stuchly, J. Microwave Power, 15 (1980) 19.

10 W.R. Adey, Physiol. Rev., 61 (1981) 435.

11 R.D. Stoy, K.R. Foster and H.P. Schwan, Phys. Med. Biol., 27 (1982) 501.

12 U. Zimmerman, Biochim. Biophys. Acta, 694 (1982) 227.

13 D.B. Kell and G.D. Hitchens, in Coherent Excitations in Biologcal Systems, H. Fröhlich and F. Kremer (Editors), Springer-Verlag, Heidelberg, 1983, p. 178.

14 G. Schwarz, J. Phys. Chem., 66 (1962) 2636.

15 C.W. Einolf and E.L. Carstensen, Biophys. J., 9 (1969) 634.

16 S.S. Dukhin and V.N. Shilov, Dielectric Phenomena and the Double Layer in Disperse Systems and Polyelectrolytes, Wiley, Chichester, 1974.

17 D.B. Kell, Biochim. Biophys. Acta, 549 (1979) 55.

18 D.B. Kell and J.G. Morris, in Vectorial Reactions in Electron and Ion Transport in Mitochondria and Bacteria, F. Palmieri, E. Quagliariello, N. Siliprandi and E.C. Slater (Editors), Elsevier/North Holland, Amsterdam, 1981, p. 339.

19 H.P. Schwan in Physical Techniques in Biological Research, W.L. Nostuk (Editor), Academic Press, New York, 1963, Vol. VIB, p. 323.

20 D. Rosen in Analytical Methods of Protein Chemistry, P. Alexander and H.P. Lundgren (Editors), Pergamon, Oxford, 1963, p. 192.

21 V.V. Daniel, Dielectric Relaxation, Academic Press, London, 1967.

22 R.G. Bennett and J.H. Calderwood, in Complex Permittivity, B.K.F. Scaife (Editor), English Universities Press, London, 1971, p. 112.

23 K. Doblhofer and A.A. Pilla, J. Electroanal. Chem., 39 (1972) 91.

24 A.A. Pilla and G.S. Margules, J. Electrochem. Soc., 124 (1977) 1697.

25 H.G.L. Coster and J.R. Smith, Biochim. Biophys. Acta, 373 (1974) 151.

26 D.J. Bell, H.G.L. Coster and J.R. Smith, J. Phys. E, 8 (1975) 66.

27 R.G. Ashcroft, H.G.L. Coster and J.R. Smith, Biochim. Biophys. Acta, 643 (1981) 191.

28 R.H. Cole, J. Phys. Chem., 79 (1975) 1459, 1469.

29 H. Nakamura, Y. Husini and A. Wada, Jpn. J. Appl. Phys., 16 (1977) 2301. 
30 C. Boned and J. Peyrelasse, J. Phys. E, 15 (1982) 534.

31 C. Gabrielli and M. Keddam, Electrochim. Acta, 19 (1974) 355.

32 A.J. Bard and L.R. Faulkner, Electrochemical Methods, Wiley, Chichester, 1980.

33 A.M. Bond, Modern Polarographic Methods in Analytical Chemistry, Marcel Dekker, New York, 1980.

34 R. Nowotny and C. Nowotny, Med. Biol. Eng. Comput., 18 (1980) 779.

35 C.P.M. Bongenaar, M. Slutyers-Rehbach and J.H. Slutyers, J. Electroanal. Chem., 109 (1980) 23.

36 L.J. Schoonman, J.R. Stil, J.R. MacDonald and D.R. Franceschetti, Solid State Ionics, 3/4 (1981) 365.

37 J.J. Almasi and O.H. Schmitt, Comput. Biomed. Res., 7 (1974) 449.

38 H.A. Kolb and G. Boheim, J. Membr. Biol., 38 (1978) 151.

39 S. Umemura, R. Hayakawa and Y. Wada, Biophys. Chem., 11 (1980) 317.

40 R.P. Scobey, Comput. Programs Biomed., 13 (1981) 3.

41 H. Nakamura, Y. Husimi and A. Wada, J. Appl. Phys., 52 (1981) 3053.

42 R.D. Tucker, T.R. Hudrlik, S.E. Silvus and E. Ackerman, Comput. Biol. Med., 11 (1981) 153.

43 D. Poussart, L.E. Moore and H.M. Fishman, Ann. N.Y: Acad. Sci., 303 (1977) 355.

44 P.Z. Marmarelis and V.Z. Marmarelis, Analysis of Physiological Systems. The White-Noise Approach, Plenum Press, New York, 1978.

45 L.J. DeFelice, Introduction to Membrane Noise, Plenum Press, New York, 1981.

46 H.M. Fishman, L.E. Moore and D. Poussart, in The Biophysical Approach to Excitable Membranes, W.J. Adelman Jr. and D.E. Goldman (Editors), Plenum Press, New York, 1981, p. 65.

47 R.E. Taylor, J.M. Fernandez and F. Bezanilla, in The Biophysical Approach to Excitable Membranes, W.J. Adelman Jr. and D.E. Goldman (Editors), Plenum Press, New York, 1981, p. 97.

48 H. Pauly and H.P. Schwan, Biophys. J., 6 (1966) 621.

49 H. Pauly, L. Packer and H.P. Schwan, J. Biophys. Biochem. Cytol., 7 (1960) 589.

50 H. Fricke and H.J. Curtis, Nature (London), 134, (1934) 102.

51 Y. Sugiura, S. Koga and H. Akabori, J. Gen. Appl. Microbiol., 10 (1964) 163.

52 K. Asami, T. Hanai and N. Koizumi, J. Membr. Biol., 28 (1976) 169.

53 K. Asami, T. Hanai and N. Koizumi, J. Membr. Biol., 34 (1977) 145.

54 K.S. Cole and R.H. Cole, J. Chem. Phys., 9 (1941) 341.

55 F.A. Grant, J. Appl. Phys., 29 (1958) 76.

56 H. Fricke and S. Morse, Phys. Rev., 25 (1925) 361.

57 R.E. De La Rue and C.W. Tobias, J. Electrochem. Soc., 106 (1959) 827.

58 H.P. Schwan, S. Takashima, V.K. Miyamoto and W. Stockenius, Biophys. J., 10 (1970) 1102.

59 A. Irimajiri, T. Hanai and A. Inouye, Experientia, 31 (1975) 1373.

60 R.D. Meredith and C.W. Tobias, J. Electrochem. Soc., 108 (1961) 286.

61 H. Pauly and L. Packer, J. Biophys. Biochem. Cytol., 7 (1960) 603.

62 W.R. Redwood, S. Takashima, H.P. Schwan and T.E. Thompson, Biochim. Biophys. Acta, 255 (1972) 557.

63 N.K. Packham, J.A. Berriman and J.B. Jackson, FEBS Lett., 89 (1978) 205.

64 D.B. Kell, S.J. Ferguson and P. John, Biochim. Biophys. Acta, 502 (1978) 111.

65 D.B. Kell, P. John and S.J. Ferguson, Biochem. J., 174 (1978) 257.

66 J.N. Strathern, E.W. Jones and J.R. Broach (Editors), The Molecular Biology of the Yeast Saccharomyces, Cold Spring Harbor Laboratory, 1981.

67 K. Asami, T. Hanai and N. Koizumi, Biophys. J., 31 (1980) 215.

68 C.M. Harris, G.D. Hitchens and D.B. Kell, Proc. Int. Symp. on Bioelectrochemistry and Bioenergetics, Nottingham, 4-9 September 1983, in press. 